\title{
Antecedentes del Éxito de los Sistemas de Planificación de Recursos Empresariales en las Grandes Empresas Chilenas: un Modelo Factorial Exploratorio
}

Sylvia M. Pinto(1), Patricio E. Ramírez ${ }^{(2) *}$ y Elizabeth E. Grandón ${ }^{(1)}$

(1) Universidad del Bío-Bío, Facultad de Ciencias Empresariales, Avenida Collao 1202, Concepción,

Chile. (e-mail: marcela@ubiobio.cl, egrandon@ubiobio.cl)

(2) Escuela de Ingeniería, Universidad Católica del Norte, Larrondo 1281, Coquimbo, Chile.

(e-mail: patricio.ramirez@ucn.cl).

${ }^{*}$ Autor a quien debe ser dirigida la correspondencia

Recibido Oct. 14, 2016; Aceptado Dic. 15, 2016; Versión final Ene. 20, 2017, Publicado Jun. 2017

Resumen

El objetivo de este estudio es determinar una estructura factorial de los antecedentes del éxito de los sistemas de Planificación de Recursos Empresariales (ERP). Se recolectaron datos de grandes empresas Chilenas. Los resultados indican que los antecedentes de éxito se pueden reducir a tres dimensiones: organización, proyecto y personas, las que explican un $74,4 \%$ de la varianza extraída y presentan altos niveles de confiabilidad. Adicionalmente, un análisis de diferencia de media indica que existen diferencias estadísticamente significativas para la dimensión proyecto, asociada a los años de operación del sistema. Por otra parte, la mayoría de los encuestados valoran todos los antecedentes como importantes para el éxito del ERP, destacando los asociados a la dimensión organización. Entre ellos destacan la existencia de una comunicación efectiva, capacitación en el uso del sistema, participación del personal, y que la implementación esté acorde con las estrategias del negocio.

Palabras clave: factores críticos de éxito; ERP; modelo factorial; grandes empresas

\section{Antecedents of Success of Enterprise Resource Planning Systems in Large Chilean Enterprises: an Exploratory Factorial Model}

\begin{abstract}
The aim of this study is to determine a factorial structure of the antecedents of successful of Enterprise Resource Planning (ERP) systems. Data were collected from large Chilean companies. The results indicate that the antecedents of success can be reduced to three dimensions: organization, project, and people, all that accounts for $74.4 \%$ of the variance extracted and have high levels of reliability. Additionally, an analysis of mean difference indicates that there are statistically significant differences for the project dimension associated with the years of system operation. Moreover, most of the participants in the study indicate that all factors are important for the success of the ERP, highlighting those associated with the organization dimension. Among them are: having effective communication, training in the use of the system, staff participation, and that implementation is consistent with business strategies.
\end{abstract}

Keywords: critical success factors; ERP; factorial model; large firms 


\section{INTRODUCCIÓN}

La literatura evidencia los impactos de los sistemas empresariales (ERP, Enterprise Resource Planning) en las organizaciones que los implementan (Aloini et al., 2007; Bueno y Salmerón, 2008; Carton y Adam, 2005; Ramírez y García, 2005a; Scott y Vessey, 2000). Entre ellos destacan beneficios tangibles e intangibles, que afectan positivamente rentabilidad, productividad, toma de decisiones, servicio al cliente, y gestión de materias primas (Grabski et al., 2011; Murphy y Simon, 2002). Por otra parte, la literatura de dirección de empresas destaca el trabajo de Rockart (1979) sobre los factores críticos de éxito (FCE). Rockart basó su trabajo en los "factores de éxito" propuestos en la concepción inicial de Daniel (1961), quien los definió como tres a seis factores que determinan el éxito de la empresa, e indicó además que las tareas asociadas a estos factores clave se deberían realizar extremadamente bien para que la empresa sea exitosa. En este contexto, muchos estudios se han centrado en determinar los FCE de la implementación de ERP. De hecho, Huang y Yasuda (2016) dan cuenta de 86 publicaciones que realizaron revisiones de literatura en el tema de ERP, de ellas, 21 se centraron en determinar los FCE en la implementación. Según estos autores, por una parte, los FCE examinados en cada estudio son numerosos, llegando algunos a más de un centenar, y por otra, la mayoría se enfoca en pequeñas y medianas empresas y en países desarrollados. Una revisión detallada de la literatura asociada a los sistemas ERP, como así una proyección de temas de investigación asociados al fenómeno puede ser encontrada en Grabski et al. (2011).

Dado el estado inicial de los sistemas ERP en países en desarrollo en relación a países desarrollados (Gupta et al., 2014), pocos estudios se han centrado en conocer los FCE en la implementación de ERP en Latinoamérica (Asemi y Jazi, 2010), lo que es acorde al desarrollo científico limitado de la región (Ramírez y Mariano, 2014). Entre estos estudios, se pueden destacar los de Colmenares (2005), De Freitas et al. (2015), Ramírez y García (2005b), Benvenuto (2006) y Ramírez y García (2007). El primero consistió en conocer las percepciones de los gerentes de proyectos de implantación de sistemas ERP en 7 grandes empresas Venezolanas, y como resultado se obtuvo una serie de FCE a considerar al momento de implementar este tipo de soluciones. Por su parte, De Freitas et al. (2015), a través de un panel de expertos identificó los criterios que se pueden utilizar para evaluar el éxito en la implementación de sistemas ERP en grandes empresas Brasileñas. De este modo, identificó 34 FCE y validó 25 utilizando el método de validez de contenido en base a la tasa de acuerdo entre los expertos (Chang et al., 2011). Los tres estudios finales de la lista destacada corresponden a investigaciones asociadas a los FCE en empresas chilenas.

Es el caso de Ramírez y García (2005b), los autores determinaron el impacto de 8 factores en el éxito de la implementación de sistemas ERP en base al modelo de DeLone y McLean (2003). Entre los principales factores destaca el compromiso ejecutivo, habilidades en tecnologías de información y en procesos de negocio, entrenamiento en ERP, aprendizaje y predisposición para el cambio. Benvenuto (2006) estudió las motivaciones que tienen las empresas para implementar sistemas ERP, los niveles jerárquicos involucrados en la decisión de implantar esta tecnología, el nivel de integración de los ERP con otros sistemas en la empresa y los beneficios esperados versus los beneficios tangibles observados. Para ello aplicó un cuestionario a 11 grandes empresas usuarias de ERP y sus principales conclusiones indican que la motivación más importante para adoptar sistemas de esta naturaleza es tener acceso a información confiable, precisa y oportuna, que durante el proceso de implantación las empresas cambian su estructura organizacional y que los ERP no se integran plenamente a proceso contables administrativos ni a otras tecnologías disponibles en el mercado. Finalmente, Ramírez y García (2007) estudiaron los factores asociados a la implementación de sistemas ERP que pueden generar una ventaja competitiva sostenible para la empresa. Basados en la teoría de recursos y capacidades (Barney et al., 2001) los autores encontraron que el aprendizaje y la predisposición para el cambio, en conjunto con otros factores, pueden ser fuente de ventaja competitiva sostenible en las empresas.

De acuerdo a la concepción inicial de FCE, y siguiendo la premisa de Daniel (1961), se hace necesario sistematizar y reducir la gran cantidad de factores encontrados en la literatura para orientar a las empresas a enfocar sus esfuerzos en áreas significativas que aseguren el éxito de la adopción de sistemas ERP. De este modo, el propósito de esta investigación es explorar los antecedentes del éxito en la implantación de sistemas ERP en grandes empresas chilenas y proponer un conjunto reducido de factores en los cuales la alta administración pueda centrarse. Para este fin, se determina la estructura factorial de los antecedentes a la implementación de sistemas ERP a través de análisis factorial exploratorio y se valoran, en forma individual, los antecedentes a la implementación de acuerdo a la importancia que entregan los directivos de las empresas. 


\section{MATERIALES Y MÉTODOS}

Este estudio se basa en métodos cuantitativos en donde se aplicó un cuestionario previamente validado para determinar el uso de sistemas ERP en las grandes empresas Chilenas. Los elementos muestréales son no probabilísticos, del tipo intencional, donde los criterios de selección de la muestra fueron a) disponibilidad de las empresas a entregar información, b) disponibilidad de tiempo de las personas que respondieron, c) facilidad de contactar a los encargados del área. Se contactó a 250 de las 500 grandes empresas listadas en el ranking publicado anualmente por la revista América Economía. El proceso para conformar la muestra consistió de 3 grandes etapas, donde primero se recopiló los contactos de las empresas, reuniendo su correo electrónico y/o teléfono general que facilitaban sus respectivas páginas web. Luego, se contactó telefónicamente a las empresas explicando el objetivo de la llamada y con esto obtener los datos de contacto de la persona con el conocimiento en el área y/o que hubiera participado del proceso de implantación de un ERP. Por último, identificadas las personas, se enviaron correos electrónicos personales explicando nuevamente la finalidad del estudio e indicando el link con la página web que contenía dicho instrumento. De las 250 empresas contactadas, mostraron interés en participar en el estudio 189 empresas, de las cuales 56 respondieron la encuesta, 113 visitaron la página web pero no respondieron y 20 no visitaron la página para completar la encuesta. Se realizó un seguimiento (telefónico o vía correo electrónico) a aquellas empresas que durante el primer mes no contestaron la encuesta.

La encuesta consta de 3 partes: información general de la empresa, información acerca del uso de sistemas ERP e información de los FCE en la implementación de sistemas ERP, con un total de 51 preguntas. Las preguntas asociadas a los FCE se midieron a través de una escala Likert de 5 puntos (muy importante a muy poco importante). El instrumento fue validado por académicos expertos en el área y posteriormente por dos empresas, una del rubro electricidad (que utiliza un ERP world class) y otra del rubro comercio (que utiliza un sistema propio). En particular, y en relación a la evaluación de las propiedades métricas de las escalas asociadas al cuestionario, se utilizó la validez de contenido para valorar el grado en que este instrumento contribuía a medir el fenómeno para el cual fue diseñado. En ese sentido, se sometió el cuestionario a la valoración de seis informantes claves, cuatro académicos expertos en ERP y dos responsables de la gestión de tecnologías de información en empresas usuarias de ERP. Se obtuvieron las valoraciones cualitativas de los informantes a través de entrevistas. Como resultado de esta validación se realizaron pequeños ajustes a las preguntas del cuestionario.

La encuesta se gestionó a través de la plataforma SURVIO, la cual permitió llevar un mejor control de visitas y respuestas del instrumento. En la Introducción se incluyó el propósito del estudio, la motivación o importancia de su participación, agradecimiento, el tiempo lo suficientemente abierto para no presionar al encuestado, identificación de quiénes lo aplican, y una cláusula de confidencialidad del manejo de la información, además de instrucciones iniciales claras y sencillas. Los FCE contemplados en el instrumento fueron 26 y se obtuvieron a partir de una revisión sistemática de la literatura (Fell et al., 2013), que fue completada con estudios desarrollados en países Latinoamericanos (Colmenares, 2005; Ramírez y García, 2005c, García y Pérez, 2006). Se solicitó a las empresas que calificaran cada uno de ellos utilizando la escala diseñada para tal fin. Se realizó un Análisis Factorial exploratorio para determinar la estructura de los factores críticos que llevan al éxito la implementación de sistemas ERP.

\section{RESULTADOS}

Las empresas encuestadas provienen de los principales sectores económicos del país, cuya distribución se muestra en la Tabla 1.

Tabla 1: Distribución según sector económico.

\begin{tabular}{lcc}
\hline Sector & Frecuencia & $\%$ \\
\hline Industria manufacturera y Servicios & 27 & 48,2 \\
Comercio & 9 & 16,1 \\
Pesca & 6 & 10,7 \\
Minería & 6 & 10,7 \\
Agropecuario-silvícola & 5 & 8,9 \\
Construcción & 3 & 5,4 \\
\hline
\end{tabular}

El $90 \%$ de las empresas encuestadas usan o están en proceso de implantación de un sistema ERP. Entre ellos, SAP es el que presenta una mayor presencia (42,9\%) seguido de Softland con un 19,6\%. La Tabla 2 , muestra el detalle de los sistemas ERP utilizados. Respecto del tiempo de uso del sistema ERP, se puede apreciar de la que el $42 \%$ de ellas lleva más de 7 años en explotación del sistema. 
Tabla 2: Sistemas ERP en uso.

\begin{tabular}{lcc}
\hline Sistema ERP & Frecuencia & $\%$ \\
\hline SAP & 24 & 42,9 \\
Otros & 16 & 28,6 \\
Softland & 11 & 19,6 \\
Sonda & 2 & 3,6 \\
Oracle & 1 & 1,8 \\
Transtecnia & 1 & 1,8 \\
Random & 1 & 1,8 \\
\hline
\end{tabular}

Tabla 3: Tiempo de uso del sistema.

\begin{tabular}{lcc}
\hline Tiempo & Frecuencia & $\%$ \\
\hline En proceso de implantación (Entre 6 y 12 meses aprox.) & 1 & 2,0 \\
Entre 1 y 3 años & 12 & 24,0 \\
Entre 4 y 7 años & 16 & 32,0 \\
Más de 7 años & 21 & 42,0 \\
\hline
\end{tabular}

La mayoría de los encuestados (45\%) indicaron que el tiempo de implementación del sistema fue entre 1 y 2 años, mientras que el 37\% de ellos indicó que tardaron menos de un año en implementar el ERP.

Tabla 4: Tiempo de implantación del sistema.

\begin{tabular}{lcc}
\hline Tiempo & Frecuencia & $\%$ \\
\hline Menos de un año & 18 & 36,7 \\
Entre 1 y 2 años & 22 & 44,9 \\
Entre 2 y 3 años & 4 & 8,2 \\
Más de 3 años & 5 & 10,2 \\
\hline
\end{tabular}

Por otra parte, es importante destacar que los módulos de Gestión Financiera, Gestión de Activo Fijo, Gestión de Materiales y Gestión de Ventas son los más implementados por las empresas. La Tabla 5 muestra la distribución detallada.

Tabla 5: Módulos implantados.

\begin{tabular}{lll}
\hline Módulo & \multicolumn{2}{c}{ Frecuencia } \\
\hline Gestión Financiera & 44 & 78,6 \\
Gestión Activo Fijo & 40 & 71,4 \\
Gestión de Materiales & 39 & 69,6 \\
Gestión Venta & 34 & 60,7 \\
Gestión Distribución y logística & 32 & 57,1 \\
Gestión de Recursos Humanos & 28 & 50,0 \\
Planificación y control de la producción & 20 & 35,7 \\
Gestión de proyectos & 16 & 28,6 \\
Otros & 10 & 17,9 \\
\hline
\end{tabular}

Respecto a la valoración de los antecedentes de éxito en la implementación de sistemas ERP efectuada por los encuestados, se observa que más del $80 \%$ consideran todos los antecedentes como muy importantes o importantes para el éxito del ERP. La Figura 1 muestra los antecedentes más valorados por las empresas (la lista de todos los antecedentes se presenta en la Tabla 7), entre ellos se encuentran "disponer de comunicación efectiva en la implantación/explotación del proyecto", "realizar capacitaciones en el uso del ERP para contar con personal mejor preparado", "contar con participación activa del personal de la empresa que va a trabajar con el ERP", y que "las estrategias de implementación estén acorde con las estrategias del negocio". Por otro lado, el factor "contar con consultores externos que evalúen el uso del ERP" es el menos valorado por los encuestados (3,57 en promedio). 


\section{Análisis Factorial Exploratorio}

Con el propósito de analizar en profundidad los antecedentes de éxito valorizados por los encuestados, se realizó un análisis factorial exploratorio. Las escalas de medida de todas las variables eran homogéneas (con mínimo 1 y máximo 5). El test de Kolgomorov-Smirnov indicó que las variables no se distribuían en forma normal por lo que para resolver este problema se procedió a tratarlas con familia de transformaciones de BoxCox. Este tratamiento mejoró la curtosis y asimetría de la mayoría de las variables, pero no logro su normalidad. La Tabla 6 indica la pertinencia de aplicar este análisis a los datos disponibles, dado que el valor de la prueba de esfericidad de Bartlett $(1.404 ; p=0,00)$ indica que la matriz de correlaciones es significativamente distinta de la matriz identidad, y que adicionalmente, el valor de Kaiser-Meyer-Olkin es superior al límite inferior admitido de 0,5.

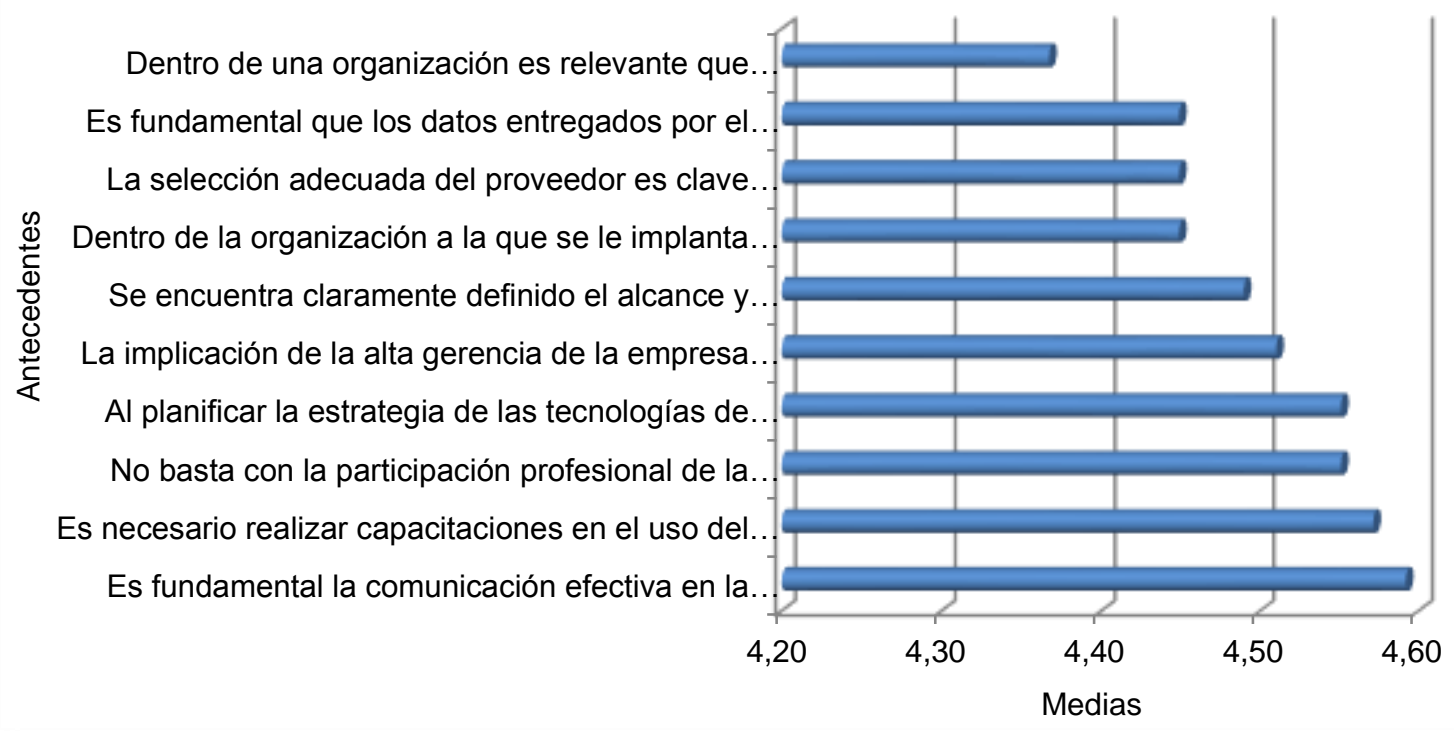

Fig. 1: Antecedentes más valorizados por los encuestados.

Tabla 6: Kaiser-Meyer-Olkin y prueba de Bartlett

\begin{tabular}{|l|l|l|}
\hline \multicolumn{2}{|c|}{ Medida de adecuación muestral de Kaiser-Meyer-Olkin } & 0,900 \\
\hline \multirow{3}{*}{ Prueba de esfericidad de Bartlett } & Aprox. Chi-cuadrado & $1.404,285$ \\
\cline { 2 - 3 } & Gl & 325 \\
\cline { 2 - 3 } & Sig. & 0,000 \\
\hline
\end{tabular}

La matriz de factores rotada después de trece iteraciones señala la existencia de tres factores (ver Tabla 7), que son los que presentan autovalores mayores que la unidad. Tras la rotación ortogonal (varimax) se procede a la interpretación de las dimensiones obtenidas: (i) La Dimensión 1 agrupa a 16 antecedentes, donde los tres primeros en carga factorial son: "es fundamental la comunicación efectiva en la implantación / explotación del proyecto", "es fundamental que los datos entregados por el sistema sean los requeridos por quien los utilice", y "la implicación de la alta gerencia de la empresa en el proyecto es fundamental para garantizar el éxito". A esta Dimensión se le denominó "Organización”; (ii) La Dimensión 2 incluye a 7 antecedentes, entre ellos los dos primeros en carga factorial son: "un buen ERP debe integrarse plenamente y de forma transparente con otros programas que se usen en la compañía" y "es necesario contar con consultores externos que evalúen el uso del ERP”. A esta Dimensión se le denominó "Proyecto"; (iii) La Dimensión 3 agrupa a 3 antecedentes, de ellos los dos primeros en carga factorial son: "es importante contar con personal que tenga habilidades en tecnologías de información" y "es fundamental que los usuarios del sistema tengan un nivel de conocimiento técnico del negocio". A esta Dimensión se le denominó "Personas". La varianza explicada por los tres factores es el 74,41\% y el Alfa de Cronbach para cada uno de los antecedentes van de 0,799 a 0,976.

\section{Análisis de Diferencias de Media}

Se realizaron pruebas de diferencias de media para las tres dimensiones detectadas en el procedimiento anterior. Primero se utilizaron como variables de agrupación el rubro, el tamaño de la empresa (medido en número de empleados), y el uso o no uso del sistema ERP, estas pruebas no indicaron diferencias estadísticamente significativas para ninguna dimensión. Por otra parte, y como se puede ver en las dos últimas 
columnas de la Tabla 7, los resultados de la prueba ANOVA indicaron diferencias estadísticamente significativas para la dimensión Proyecto, entre las empresas que implementaron el sistema ERP en tres años o más y aquellas empresas que lo implementaron en un plazo menor. Como las variables no cumplieron con el requisito de normalidad, se aplicaron técnicas no paramétricas (Mann-Whitney y Kolmogorov-Smirnov), para corroborar los resultados de ANOVA. Según estos resultados, si bien las dimensiones Organización y Personas no muestran diferencias estadísticamente significativas en función al tiempo de implementación del ERP, la dimensión Proyecto es un $23 \%$ más importante para aquellas organizaciones que han implantado el ERP en menos tiempo.

Tabla 7: Matriz de factores rotados

\begin{tabular}{|c|c|c|}
\hline \multicolumn{2}{|r|}{ Dimensión 1: Organización (Alfa de Cronbach = 0,976) } & Carga \\
\hline A3 & Es fundamental la comunicación efectiva en la implantación / explotación del proyecto. & 0,818 \\
\hline A25 & Es fundamental que los datos entregados por el sistema sean los requeridos por quien los utilice. & 0,805 \\
\hline A1 & $\begin{array}{l}\text { La implicación de la alta gerencia de la empresa en el proyecto es fundamental para garantizar el } \\
\text { éxito. }\end{array}$ & 0,796 \\
\hline A18 & Es necesario realizar capacitaciones en el uso del ERP para contar con personal mejor preparado. & 0,795 \\
\hline A23 & $\begin{array}{l}\text { Al planificar la estrategia de las tecnologías de información es fundamental que estas estén acorde } \\
\text { con la estrategia del negocio. }\end{array}$ & 0,786 \\
\hline $\mathrm{A} 2$ & Se encuentra claramente definido el alcance y metas del proyecto. & 0,782 \\
\hline A13 & $\begin{array}{l}\text { Dentro de la organización a la que se le implanta el ERP, debe existir un responsable de proyecto } \\
\text { ERP con poder de decisión. }\end{array}$ & 0,764 \\
\hline A5 & $\begin{array}{l}\text { No basta con la participación profesional de la empresa implantadora, es también necesaria la } \\
\text { participación activa del personal de la empresa que va a trabajar con el ERP. }\end{array}$ & 0,736 \\
\hline A16 & La selección adecuada del proveedor es clave para garantizar el éxito. & 0,718 \\
\hline A6 & $\begin{array}{l}\text { Es fundamental contar con un estándar y ser disciplinario en el desarrollo de los procedimientos de } \\
\text { implantación. }\end{array}$ & 0,715 \\
\hline A24 & $\begin{array}{l}\text { Muchas veces la implantación de un ERP implica la necesidad de una gestión del cambio que la } \\
\text { empresa, futura usuaria del ERP, debe asumir y comprender. }\end{array}$ & 0,699 \\
\hline A8 & Dentro de una organización es relevante que estas tengan claridad de los objetivos de proyecto. & 0,668 \\
\hline A12 & $\begin{array}{l}\text { Es necesario que el proveedor cuente con las herramientas necesarias para dar solución a los } \\
\text { requerimientos del cliente. }\end{array}$ & 0,635 \\
\hline A4 & Dentro de las organizaciones que se implantan ERP debe existir un comité de dirección del proyecto. & 0,579 \\
\hline A9 & La comunicación entre departamentos es clave para que la implementación del ERP sea exitosa. & 0,575 \\
\hline A17 & $\begin{array}{l}\text { Es necesario contar con las herramientas computarizadas necesarias para el buen desarrollo de un } \\
\text { proyecto. }\end{array}$ & 0,530 \\
\hline \multicolumn{3}{|c|}{ Dimensión 2: Proyecto (Alfa de Cronbach = 0,912) } \\
\hline A15 & $\begin{array}{l}\text { Un buen ERP debe integrarse plenamente y de forma transparente con otros programas que se usen } \\
\text { en la compañía. }\end{array}$ & 0,726 \\
\hline A19 & Es necesario contar con consultores externos que evalúen el uso del ERP & 0,714 \\
\hline A7 & $\begin{array}{l}\text { Es clave para el éxito del proyecto ERP tener definidas desde un principio la estrategia de } \\
\text { implantación. }\end{array}$ & 0,659 \\
\hline A21 & $\begin{array}{l}\text { Es fundamental contar con un plan de contingencias y de previsión de riesgos durante el proyecto } \\
\text { ERP. }\end{array}$ & 0,623 \\
\hline A26 & $\begin{array}{l}\text { Es importante que el proveedor de ERP monitoree y evalúe constantemente el desempeño del } \\
\text { sistema. }\end{array}$ & 0,619 \\
\hline A20 & $\begin{array}{l}\text { Tan importante o más que el propio proceso de implantación es el soporte y mantenimiento } \\
\text { especializado disponible durante el uso del ERP. }\end{array}$ & 0,553 \\
\hline A14 & $\begin{array}{l}\text { El ERP debe estructurarse según el trabajo que realizan las personas de la organización y cubrir } \\
\text { todas las funciones y roles necesarios. }\end{array}$ & 0,541 \\
\hline \multicolumn{3}{|c|}{ Dimensión 3: Personas (Alfa de Cronbach =0,799) } \\
\hline A22 & Es importante contar con personal que tenga habilidades en tecnologías de información. & 0,763 \\
\hline A11 & Es fundamental que los usuarios del sistema tengan un nivel de conocimiento técnico del negocio. & 0,674 \\
\hline A10 & Es importante contar con la participación activa de los usuarios finales en la implantación de un ERP. & 0,641 \\
\hline
\end{tabular}

Tabla 8: Prueba ANOVA

\begin{tabular}{|l|c|c|c|c|c|c|}
\hline & \multicolumn{2}{|c|}{$\begin{array}{c}\text { Tres o más años de } \\
\text { implementación( } N=8)\end{array}$} & $\begin{array}{c}\text { Menos de tres años de } \\
\text { implementación }(N=40)\end{array}$ & \multicolumn{3}{c|}{ ANOVA } \\
\hline Factor & Media & $\begin{array}{c}\text { Desviación } \\
\text { estándar }\end{array}$ & Media & $\begin{array}{c}\text { Desviación } \\
\text { estándar }\end{array}$ & $\mathrm{F}$ & Sig. \\
\hline Organización & 3,86 & 1,61 & 4,50 & 0,66 & 3,67 & 0,062 \\
\hline Proyecto & 3,38 & 1,39 & 4,15 & 0,68 & 5,84 & 0,020 \\
\hline Personas & 3,50 & 1,47 & 4,01 & 0,73 & 2,23 & 0,142 \\
\hline
\end{tabular}




\section{CONCLUSIONES}

Los FCE inciden en un exitoso proceso de implementación de ERP. Sin embargo, el gran número de estos factores indicados en la literatura torna inmanejable que una organización se centre en aquellos que podrían tener un mayor impacto. Los resultados de este estudio reducen el número de estos factores a tres dimensiones: Organización, Proyecto y Personas. Estas dimensiones agrupan a los antecedentes estudiados y contribuyen a enfrentarse a procesos de implantación de ERP en forma más parsimoniosa. La Organización es la dimensión que explica una mayor varianza en los antecedentes, y coincidentemente, incluye aquellos ítems más valorados por los encuestados.

El análisis entre las tres dimensiones concluye que las empresas que valoran más los antecedentes asociados a la dimensión Proyecto demoran menos en implementar el sistema ERP. Este resultado complementa la evaluación de éxito de ERP propuesta en la literatura, que no considera entre los criterios de evaluación de éxito los años de implantación. En específico, las empresas que han implementado en menos tiempo valoran más 1) contar con un plan de contingencias y previsión de riesgos, 2) tener definida desde un principio la estrategia de implantación, y 3) contar con que el proveedor del ERP monitoree constantemente el desempeño del sistema. Por otra parte, en la dimensión Organización, los antecedentes específicos más valorados son el disponer de comunicación efectiva en la implantación del proyecto y el realizar capacitaciones en el uso del ERP, lo que sugiere a las empresas la necesidad de definir y gestionar un plan de capacitación y un plan de comunicación que estén dirigidos a apoyar la aceptación y uso de este tipo de sistemas por parte de los empleados.

En general, futuros estudios deberían abordar la posible replicabilidad de estos hallazgos en una muestra mayor de organizaciones, como en otras tecnologías de información de apoyo a las empresas. También podrían explorar la causalidad entre las dimensiones encontradas en esta investigación. En particular, líneas de trabajo asociadas a los resultados específicos de este estudio podrían centrarse en determinar los elementos claves del plan de comunicación que permitan disminuir la resistencia al cambio y promover la participación de los empleados impactando directamente en el éxito de implantación de un sistema. Asimismo, la definición del tiempo, momento y formas de abordar la capacitación son temas que deben ser evaluados para establecer pautas que permitan finalmente disminuir el tiempo de implantación de este tipo de sistemas.

\section{REFERENCIAS}

Aloini, D., Dulmin, R., and Mininno, V., Risk management in ERP project introduction: review of the literature, Information \& Management, 44(6), 547-567 (2007)

Asemi, A., y Jazi, M. D., A comparative study of critical success factors (CSFs) in implementation of ERP in developed and developing countries, International Journal of Advancements in Computing Technology, 2(5), 99-110 (2010)

Barney, J., Wright, M., y Ketchen, D.J. Jr., The resource-based view of de firm: Ten years after 1991, Journal of Management, 27(6), 625-641 (2001)

Bueno, S., y Salmerón, J. L., TAM-based success modeling in ERP, Interacting with Computers, 20, 515-523 (2008)

Carton, F., y Adam, F., Understanding the impact of enterprise systems on management decision making: an agenda for future research, The Electronic Journal of Information Systems Evaluation, 8(2), 99-106 (2005)

Chang, S., Yu, S., Ng, C.S., Chang, I., y Yen, D.C., An ERP system performance assessment model development based on the balanced scorecard approach, Information Systems Frontiers, 13(3), 429-450 (2011)

Colmenares, L. E., Un estudio exploratorio sobre los factores críticos de éxito en la implantación de sistemas de planeación de recursos empresariales (ERP) en Venezuela, Journal of Information Systems and Technology Management, 2(2), 167-187 (2005)

Daniel, D.R., Management Information Crisis, Harvard Business Review, 111-122 (1961)

De Freitas, R., Costa, H., Pereira, V., y Shimoda, E., Criteria selection for evaluation of ERP systems implementation in large Brazilian companies, Management Research, The Journal of the Iberoamerican Academy of Management, 13(2), 160-186 (2015)

DeLone, W. H., McLean, E. R., DeLone and McLean Model of Information Systems Success: A Ten-Year Update, Journal of Management Information Systems, 19(4), 9-30 (2003) 
Fell, A., Pinto, M., y Rodríguez A., Definición de un conjunto de guías de adopción de un ERP basado en factores críticos de éxito, metodologías de adopción y tamaño de la organización: una revisión sistemática de la literatura, Actas de la Conferencia Infonor, Coquimbo, Chile, 12-14 agosto (2013)

García, N., y Pérez, L., Determinación de Factores Críticos de Éxito en el proceso de implementación de un sistema ERP. Un estudio de campo en empresas mexicanas, Proceedings of Americas Conference on Information Systems (AMCIS), 4342-4354 (2006)

Grabski, S.V., Leech, S. A., y Schmidt, P.J., A review of ERP Research: A future agenda for accounting information systems, Journal of Information Systems, 25(1), 37-78 (2011)

Gupta, H., Aye, K. T., Balakrishnan, R., Rajagopal, S. y Nguwi, Y.Y., Formulating, implementing and evaluating ERP in small and medium scale industries, International Journal of Advances in Computer Science and Technology, 3(6), 386-389 (2014)

Huang, T., y Yasuda, K., Comprehensive review of literature survey articles on ERP, Business Process Management Journal, 22(1), 2-32 (2016)

Murphy, K., y Simon, S.J., Intangible benefits valuation in ERP projects, Information Systems Journal, 12(4), 301-320 (2002)

Ramírez, P., y García, R., Meta-analysis on the implementation of ERP systems, Journal of Information Systems and Technology Management, 2(3), 245-273 (2005a)

Ramírez, P., y García, R., Investigación empírica sobre los factores que afectan el éxito de los sistemas ERP en Chile, Ingeniería Informática, (11), 1 (2005b)

Ramírez, P., y García, R., Success of ERP systems in Chile: an empirical study. Proceedings of European, Mediterranean \& Middle Eastern Conference on Information Systems, 1-16, El Cairo, Egipto, 7 y 8 de Junio (2005c)

Ramírez, P., y García-Cruz, R., Tecnología de información y ventaja competitiva: El caso de los sistemas ERP en Chile, Revista Electrónica de Ciencia Administrativa, 6(1), 1 (2007)

Ramírez, P., y Mariano, A., La Literatura Científica en Ciencias Empresariales: un Análisis Comparativo entre Chile y Brasil, Información Tecnológica, 25(6), 157-162 (2014)

Rockart, F., Chief executives define their own data needs, Harvard Business Review, 81-95 (1979)

Scott, J.R., y Vessey, I., Implementing enterprise resource planning systems: The role of learning from failure, Information Systems Frontiers, 2(2), 213-232 (2000) 